\title{
Instrumentación, Control y Telesupervisión en Centrales Térmicas de Pequeña Potencia.
}

\author{
Ulises Manassero ${ }^{1}$, José Luis Torres ${ }^{1}$, Diego López ${ }^{1}$, Rodrigo Furlani ${ }^{1}$, Raúl \\ Regalini ${ }^{1}$, Matias Orué ${ }^{1}$ \\ ${ }^{1}$ Laboratorio de Sistemas de Control, Universidad Tecnológica Nacional, Facultad Regional \\ Santa Fe
}

\begin{abstract}
Resumen. La empresa Energía Argentina S. A. (ENARSA) cuenta con centrales termoeléctricas distribuidas en todo el país con el fin de disminuir los problemas energéticos. Actualmente, la empresa cursa un proceso de mejora en la gestión del sistema de transporte, control del stock y consumo de combustible. El Laboratorio de Sistemas de Control (LSC) de la UTN Regional Santa Fe colabora activamente en este proceso y propone en este trabajo la adecuación de las centrales, para poder integrarlas a un sistema de Control Supervisor y Adquisición de Datos (SCADA) de administración y gestión de combustible. Contribuyendo de este modo a lograr una máxima eficiencia en las operaciones de las centrales eléctricas asegurando la optimización en la utilización de los recursos disponibles.. Este sistema toma el nombre de Sistema Integral de Supervisión (SIS). Los criterios de diseño y selección de la arquitectura de la red de comunicaciones, la instrumentación y los sistemas de control correspondientes a las CT propuestas en este trabajo, otorgan al sistema de telesupervisión una amplia flexibilidad y posibilidades de expansión.
\end{abstract}

Keywords: ENARSA, Combustible, SCADA, Centrales Térmicas.

\section{Introducción}

El sector energético argentino se encuentra actualmente inmerso en una crisis estructural de una profundidad insospechada. Si bien se están ejecutando obras importantes en el área de transmisión de energía con el propósito de mejorar la confiabilidad y estabilidad de las tensiones del sistema interconectado de 500 [kV], el incremento sostenido de la demanda desde el año 2003 a tasas anuales promedio del $5 \%$ [1] implican la necesidad de una constante repotenciación del sistema (1 [GW] por año).

Por otra parte, en el corto plazo no se vislumbra la puesta en funcionamiento de nuevas centrales eléctricas de gran potencia que puedan atender los requerimientos energéticos existentes.

Como solución inmediata a esta crisis energética, el estado nacional promueve la instalación de centrales de generación distribuida. Para ello, a partir del año 2008, la Secretaría de Energía de la Nación, encomendó a ENARSA la instalación de centrales 
térmicas (CT) de pequeña potencia (menores a 40 [MVA]) con turbogeneradores de alimentación dual -gas natural y diesel- y moto-generadores alimentados con combustible diesel.

Dichas centrales forman parte de los proyectos "Generación Distribuida I" y "Generación Distribuida II" llevado a cabo por la empresa (ver fig. 1).

Es importante destacar que la generación distribuída conlleva una serie de beneficios, dentro de los cuales se destacan los siguientes [2]:

- Reducción de la utilización de las redes de transporte de energía y de las pérdidas globales del sistema por kWh consumido efectivamente.

- Reducción de la necesidad de inversión en activos fijos en las redes de transporte de energía.

- Reducción de las pérdidas en las redes de distribución de energía.

- Incremento de la confiabilidad en el suministro de energía eléctrica.

- Posible uso para la regulación de tensión en distribución.

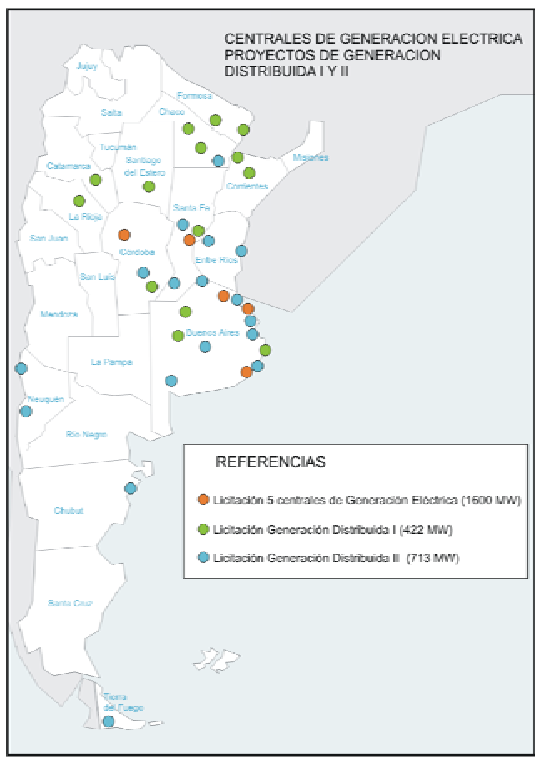

Fig. 1. Centrales térmicas de los proyectos de generación distribuída I y II ${ }^{1}$

Por otra parte, este tipo de centrales se han convertido en el modo dominante de la nueva generación eléctrica en los EE.UU. y en el resto del mundo. Lo anterior es atribuible al menor costo de instalación por kilowatt generado, a los programas de construcción más cortos y costos de operación competitivos [3].

En la actualidad, las CT modernas, para optimizar y controlar sus procesos, demandan inexorablemente sistemas SCADA que otorguen flexibilidad y facilidad de operación $[4,5]$ e instrumentación de campo dotada de sensores y actuadores seguros y confiables, configurables remotamente desde una sala de supervisión. Se requiere además implantar estrategias de control y automatización, basadas en técnicas

\footnotetext{
${ }^{1}$ Información suministrada por la empresa ENARSA
} 
convencionales y modernas, que incluyan arranques y paros completamente automáticos, activando protecciones para evitar sobre-temperaturas y sobre-presiones, entre otros aspectos relevantes [6].

En un nivel superior de desarrollo tecnológico, existen centrales térmicas con control automático de generación [7] que trabajan con técnicas de control basadas en fuzzy logic [8] y rule based [9] y redes neuronales [10], entre otras, que representan un incremento notable en la eficiencia y optimización de los recursos, tiempos de respuesta flexibilidad y confiabilidad en su operación.

Ante estas tendencias mundiales, ENARSA carece de un sistema de Control Supervisor y de Adquisición de Datos (SCADA) en las CT que se encuentran bajo su administración. Esto trae aparejado inconvenientes para realizar las programaciones de entrega de combustible, determinar la energía generada, evaluar tendencias, determinar el estado operativo de las CT y la confiabilidad de las mismas, controlar el stock de combustible y calcular el rendimiento energético (heat-rate) de los grupos generadores, entre otros.

En el presente trabajo se desarrolla la propuesta diseñada por el Laboratorio de Sistemas de Control (LSC), perteneciente al grupo de investigación CYSE (Control y Seguridad Eléctrica) - UTN -FRSF, ante las necesidades planteadas por la empresa. A tal efecto se propone implementar un Sistema Integral de Supervisión (SIS) para las centrales, con el principal objetivo de monitorear en tiempo real los siguientes parámetros: variables físicas del sistema de combustible, variables eléctricas de los grupos generadores, gráficos de tendencias, resumen de eventos, resumen de alarmas, resumen de históricos y logs de eventos y errores. El laboratorio posee una amplia experiencia en el estudio, desarrollo y aplicación de sistemas de SCADA en áreas extensas $[11,12]$.

\section{Análisis de los Requerimientos Impuestos por la Empresa Proveedora del Combustible}

ENARSA debe cumplir con los procedimientos (ej. PT 26) establecidos por la empresa CAMMESA (Compañía Administradora del Mercado Mayorista Eléctrico Sociedad Anónima). Parte de estos procedimientos se basan en una gestión del combustible eficiente, garantizando los controles que impidan errores, fraudes, o malversación del mismo. Además las normativas hacen hincapié en la implementación de un sistema de adquisición de datos en tiempo real que reporte directamente las variables físicas relevantes del sistema de medición de combustible, desde las centrales a ENARSA.

No obstante, ENARSA presenta actualmente ciertas dificultades para gestionar el sistema de transporte, control de stock y consumo de combustible, debido principalmente a las siguientes causas:

- El combustible líquido se traslada mediante camiones cisternas desde las refinerías hasta las CT. ENARSA es responsable de la custodia del combustible desde la carga en refinerías, hasta su descarga en las CT.

- Los controles de carga en refinería y de descarga en las CT se basan en mediciones de caudales volumétricos y densidades del producto. 
- El combustible líquido despachado en las CT se almacena en uno o más tanques principales. Excepto muy pocos casos, la medición de estos tanques se realiza mediante niveles de vidrio, sistemas de boya- roldana, o inmersión de varillas de nivel. Esto implica que la medición del stock de combustible tenga asociada una incertidumbre elevada.

- El stock de combustible informado a ENARSA se realiza por carga manual de planillas en las CT. No hay implementado un sistema de información en tiempo real.

- Existen elevados costos asociados a la inspección de la carga y descarga de combustible y a los faltantes entre el combustible despachado en la refinería y el recibido en las CT.

En base al análisis de los requerimientos impuestos por la reglamentación y las dificultades que surgen del mismo, el LSC propone: diseñar e implementar una red de comunicaciones de tipo industrial para instalar en CT de generación distribuida, con la finalidad de conectar distintos procesos de aplicación de modo de asegurar la explotación del SIS; elaborar las especificaciones técnicas generales de la instrumentación requerida para mejorar la precisión en la medición de las variables del sistema de control de combustible de la CT.

\section{SISTEMA INTEGRADO DE SUPERVISIÓN}

La instalación adecuada del SIS permite, por un lado, que los operadores de la CT posean en forma centralizada la información relativa a los distintos subsistemas de la misma y por otro que esa información pueda ser transmitida al Centro de Operación de Generadores (COG) de ENARSA, desde el cual se podrá monitorear el estado y funcionamiento de las CT. Los beneficios que trae aparejado pueden resumirse en: reducción de los costos de inspección, disminución de las diferencias de combustibles y eliminación de la incertidumbre del stock de combustible.

Por otra parte, el SIS se encuentra circunscrito a los límites físicos de cada una de las CT.

La implementación del SIS en las CT abarca los siguientes subsistemas:

- $\quad$ Sistema de medición de combustibles (SMC).

- $\quad$ Sistema de medición de energía.

- Tableros de control.

- Comunicaciones.

- Sistema de monitoreo de parámetros ambientales y polución.

- $\quad$ Sistema de seguridad de acceso.

- $\quad$ Sistema de visión de campo.

No obstante, en este trabajo se describirá en detalle solo los subsistemas de SMC y de comunicaciones. 


\section{INSTRUMENTACIÓN DEL SISTEMA DE MEDICIÓN DE COMBUSTIBLE}

La configuración y el tipo de instrumentos de medición seleccionados se fundamentan sobre los siguientes criterios:

- Optimizar el instrumental a instalar en las CT, basado en una simplificación que permita, por un lado, reducir los costos de adquisición y por otro aumentar la operatividad del sistema.

- Los instrumentos deben respetar los requerimientos de precisión que la empresa proveedora del combustible solicita.

- Tener en cuenta como alternativa la implementación de instrumentos de medición multivariables, de modo que permitan reducir la cantidad de instrumentos y con ello los costos de adquisición, instalación, operación y mantenimiento asociados a los mismos.

\subsection{Descripción}

El SMC tiene como fin primario la determinación adecuada de los consumos reales de combustible y de los costos asociados a los mismos. Para ello debe medir, registrar y transmitir todos los parámetros físicos del combustible (gasoil y/o gas natural) utilizado por las CT, con la precisión y frecuencia requerida.

Llevar a cabo esto no sólo implica cumplir con los requerimientos de información que las normativas de la empresa propietaria del combustible exigen, sino que además es necesario lograr una utilización eficiente del mismo.

\subsection{Arquitectura del SMC líquido}

En la figura 2, se muestra el esquema integral del SMC. Desde los tableros de E/S distribuidas, donde llegan los buses, se llevan las señales al Tablero de Control (TC), que posee un PLC que concentra todas las variables de campo de la CT. 


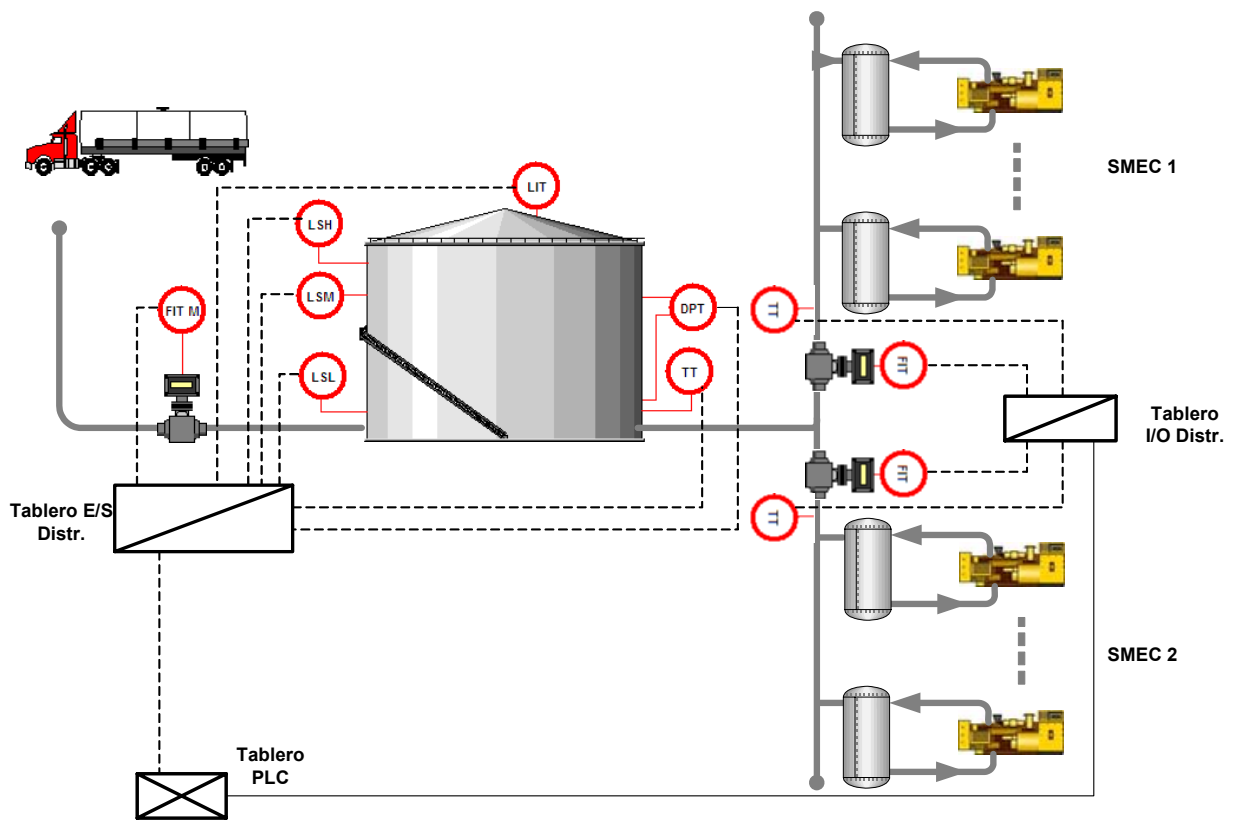

Fig. 2. Arquitectura del SMC de gas-oil

\subsection{Subsistemas del SMC líquido}

El LSC desarrolló un esquema de control, medición y telesupervisión del SMC caracterizado por su alta precisión, condiciones seguras de operación e innovación en su configuración. La operación del SMC se encuentra dividido en cuatro subsistemas a saber: a) playa de descarga de combustible; b) tanques principales; c) tanques diarios y d) grupos generadores agrupados por cada uno de los sistemas de medición de energía comercial (SMEC).

A continuación se describen cada uno de estos subsistemas.

\subsubsection{Playa de descarga de combustible.}

La playa de descarga es el lugar físico donde se realiza la descarga de los camiones cisterna que proveen de combustible a la CT. En este punto se deberá instalar un caudalímetro másico (FIT M).

La utilización de caudalímetros másicos en lugar de volumétricos tiene asociadas las siguientes ventajas:

- Se obtienen de un mismo dispositivo las variables de caudal másico, densidad y temperatura permitiendo identificar casos de adulteración de combustible.

- La variación de la densidad del combustible, y con ello el caudal volumétrico, se encuentra íntimamente relacionada con la variación de la temperatura de dicho combustible. Por ello, resulta válido afirmar que la medición del caudal volumétrico de gasoil puede incorporar grandes errores dado que a mayor 
temperatura del mismo, su densidad disminuye, incrementándose las sustancias volátiles que conllevan a un aumento del caudal volumétrico del combustible y por ende a errores en su cuantificación, o a tener la obligación de medir, de modo separado, la densidad para realizar las correcciones correspondientes. El uso de caudalímetros másicos en reemplazo de los volumétricos, permite la medición correcta del caudal de combustible dado que permite la medición de densidad, corrigiendo en forma instantánea, la cantidad de masa de combustible, por unidad de tiempo que circula a través de la cañería.

- Los costos del caudalímetro másico en comparación con el uso de caudalímetro volumétrico, donde se debe asociar un sensor de temperatura a la medición son del mismo orden.

- Su precisión ronda en 0,2\%, superando ampliamente los requerimientos de la normativa correspondiente a la empresa propietaria del combustible.

El combustible descargado es almacenado en uno o más tanques principales existentes en la central.

\subsubsection{Tanques de almacenamiento principal.}

Los tanques de almacenamiento principal son depósitos utilizados para el almacenamiento de combustible de la CT en primera instancia, luego de la descarga del camión cisterna. La instrumentación a instalar en estos tanques (ver figura 3) constará de:

- Medición continua de nivel a partir de un transmisor de nivel (LIT) que permita conocer el stock de combustible de la CT.

- Detección de nivel alto a partir de un sensor de nivel (LSH) que cumpla la función de seguridad frente a posibles rebalses de los tanques.

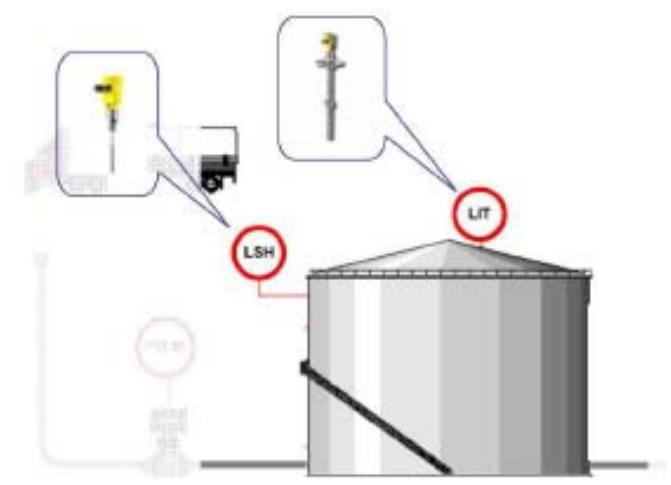

Fig. 3. Instrumentación de tanques principales

4.4.3 Tanques de almacenamiento diario y/o individual.

Los tanques de almacenamiento diario son depósitos utilizados para la acumulación de combustible de la CT en segunda instancia luego de ser trasvasado 
desde los tanques de almacenamiento principal, pasando previamente a través de un sistema de filtrado con el objetivo de eliminar impurezas sólidas y agua.

Los tanques de almacenamiento individual son depósitos, generalmente de pequeño volumen, que forman parte del conjunto motogenerador. La capacidad de los mismos depende de la autonomía propia del grupo.

Según la topología de la CT existen tanques diarios o individuales, así como también pueden coexistir ambos tipos de tanques.

La instrumentación a instalar en los tanques diarios constará de un sensor de LSH (figura 4), con el objeto de evitar posibles rebalses en dichos tanques.

Por otra parte, la instrumentación a instalar en los tanques individuales, estará compuesta por un sensor de nivel (o dos sensores, según las características del tanque) que permita detectar la condición de LSH y la condición de nivel bajo (LSL) con el fin de gobernar el automatismo de llenado de dichos tanques.

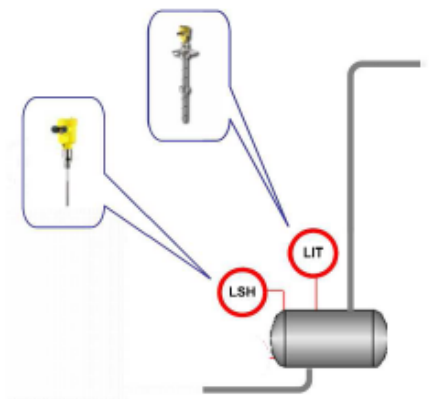

Fig. 4. Instrumentación de tanques diarios

\subsubsection{Grupos generadores agrupados por SMEC.}

En cada nodo SMEC de la CT, se determinó la instalación de caudalímetros (figura 5) para la medición del caudal másico, con el objetivo de cumplir con las exigencias de las normativas correspondientes a la empresa propietaria del combustible. Tales requisitos contemplan la obtención de información referente al caudal consumido por el conjunto de grupos generadores que conforman dicho nodo.

A partir del conocimiento de este caudal y la medición de energía generada por el nodo se obtiene el "Heat Rate" del mismo. 


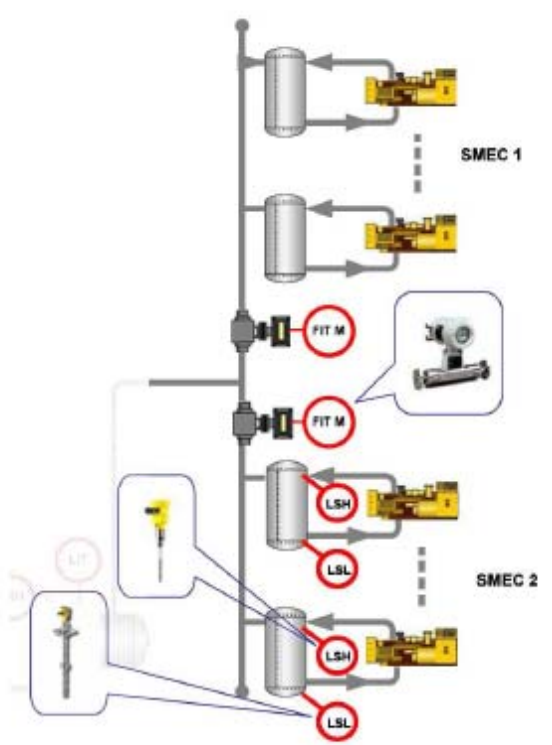

Fig. 5. Instrumentación de grupos SMEC

\section{DISEÑO DE LA RED DE COMUNICACIONES DEL SIS}

La confección de la red de comunicaciones a implementar en las CT de ENARSA demandó un estudio detallado de las diferentes tecnologías y protocolos, de modo de escoger aquella configuración que permita lograr una aceptable performance de las comunicaciones (B.E.R. ${ }^{2}$ y disponibilidad) entre los diversos buses de campo y el controlador de cada una de las CT. En este aspecto, es importante destacar que los principales requisitos que hacen a la calidad de un sistema de comunicaciones quedan establecidos a través de los siguientes parámetros [13]:

- Alta integridad y seguridad.

- Tiempo de respuesta rápida.

- $\quad$ Expandible/extensible y de fácil mantenimiento.

El trabajo llevado a cabo por LSC consistió, en una primera etapa en analizar las diferentes CT con el fin de investigar la topología de red más adecuada a implementar. Este análisis permitirá encontrar aquél diseño que sea flexible y que solucione las diferentes problemáticas encontradas en los sistemas de control y comunicaciones de las CT (ej. instrumentos sin vinculación a redes y variedades de protocolos de comunicación en una misma $\mathrm{CT}$ ).

En una segunda etapa, se definieron los equipos y dispositivos a incorporar a la red de comunicaciones ya seleccionada, con la premisa de permitir la fácil escalabilidad y una alta disponibilidad del sistema.

${ }^{2}$ BER, bit error rate (grado de error). Medida de calidad de los circuitos, expresado como el grado de bits recibidos con error sobre el número total de bits. 
A continuación se describe la topología de la red adoptada y aquellos elementos y dispositivos de comunicaciones y control que deben incorporarse al sistema.

\subsection{Topología de la red de comunicaciones}

La topología de los sistemas de comunicaciones y control para CT de generación distribuida diseñada permite lograr entre otros aspectos, escalabilidad, una baja tasa de falla, un reducido tiempo de retardo y una aceptable velocidad de transferencia de datos $\left(10^{-3} \mathrm{seg}\right)$ de las comunicaciones entre los diversos buses de campo y el PLC de control de cada una de las CT que conforman los SIS. La red implementada utiliza topologías físicas en estrella y en bus.

La topología estrella se aplica en la comunicación del PLC de control con aquellos buses de campo que tienen entradas analógicas. El PLC cumple el papel de concentrador de las señales de campo. En la figura 6 se observa la aplicación de esta topología en la comunicación de los sensores de nivel continuo (LIT) y detectores de nivel (LSL/LSH) instalados en los tanques de almacenamiento de combustible.

La topología bus se impone como el tipo de red más adecuada a implementar sobre aquellos buses de campo que soportan protocolo MODBUS (permite la comunicación física RS485 o Ethernet). El protocolo MODBUS con enlace físico RS485 se utiliza en la comunicación del PLC de Control con los medidores de energía comercial, los caudalímetros másicos y la estación meteorológica. Por otra parte, el protocolo MODBUS sobre TCP/IP establece en las CT la comunicación del PLC de control con los módulos de E/S distribuidas, la interfaz hombre máquina (HMI - por sus siglas en ingles -) y el Gateway del COG.

Es importante destacar que la combinación de ambas topologías mejora la performance del sistema de comunicaciones, que se traduce en el aumento de su confiabilidad y flexibilidad. 


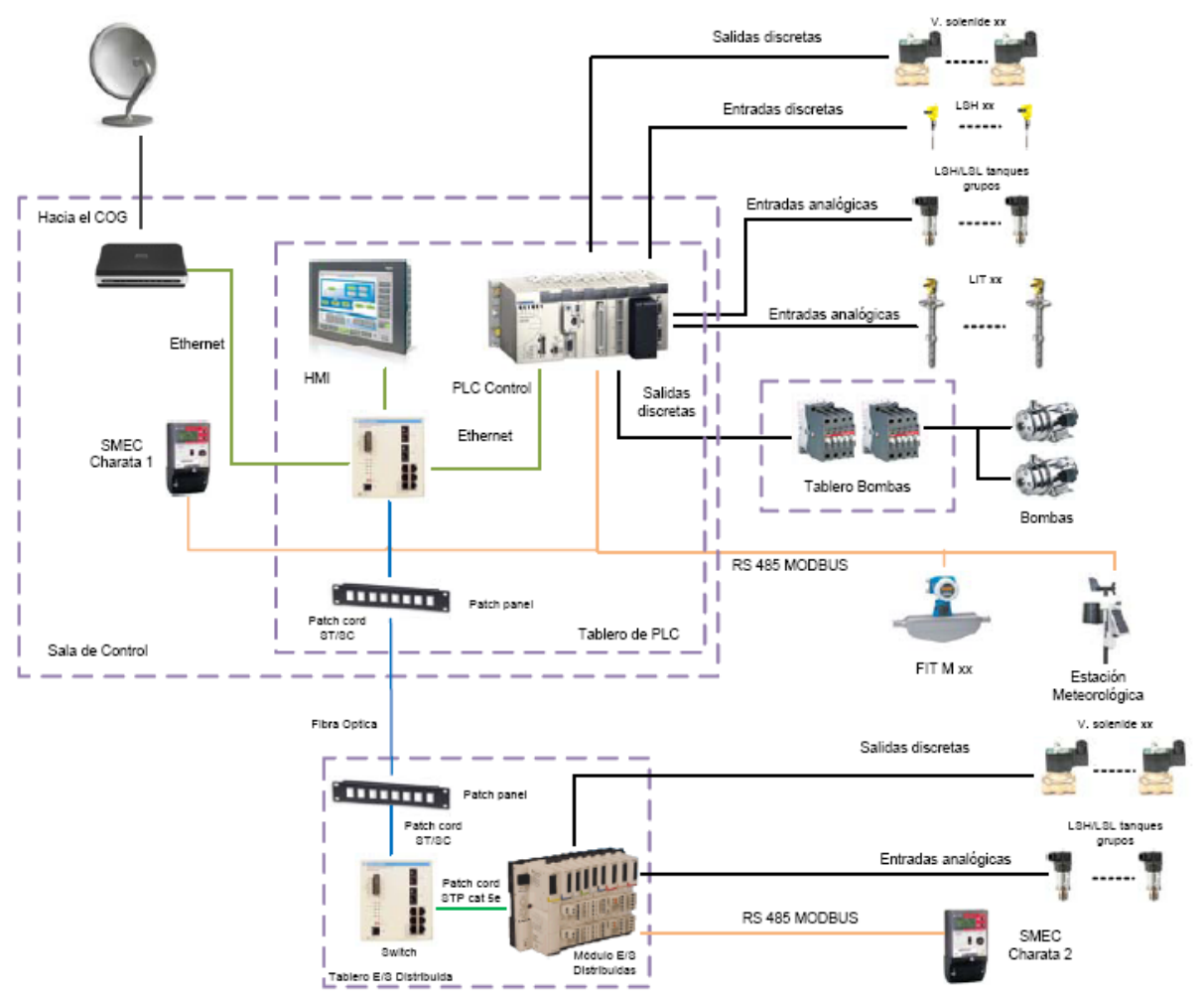

Fig. 6. Arquitectura de comunicaciones del SIS

La comunicación Ethernet industrial fue escogida como la principal red de comunicación a utilizar en las CT. Las ventajas comparativas de Ethernet TCP/IP frente a otras tecnologías de comunicación son las siguientes:

- Robustez, redundancia y durabilidad que permiten a los dispositivos seguir conectados a pesar de las condiciones agresivas en que a menudo se trabaja en la CT.

- Todos los dispositivos de E/S pueden trabajar con la web.

- La versión industrial de Ethernet supera a los viejos protocolos alternativos, casi siempre en velocidad de los componentes y el cableado.

- Reducción del costo de hardware.

- Elevado rendimiento y alta velocidad de transmisión.

- Diferentes soportes físicos de transmisión (par trenzado, cable triaxial, FO).

- Gran estandarización, independiente de fabricantes (IEEE).

- Fácil escalabilidad (10/100/1000/10000 Mbps). 


\subsection{El Tablero de Control}

De acuerdo con la configuración del esquema de control y comunicaciones propuestos, cada una de las centrales generadoras tendrá asociado a su SIS un TC. El mismo tiene por objeto recolectar toda la información perteneciente a los distintos sistemas (SMC, medición de energía, estación meteorológica). Es decir que oficiará como concentrador de todas las señales de comunicación provenientes de los diversos buses de campo, convirtiéndolo en el punto neurálgico del sistema de comunicaciones. La información recabada por el TC será transmitida al COG a través del enlace de comunicaciones suministrado por ENARSA.

Las CT se comunican con el COG vía satélite, a través de un Gateway. El PLC oficiará de vínculo entre las señales de campo y la comunicación satelital. A su vez, el COG establecerá un enlace de comunicación con CAMMESA (ver figura 7).

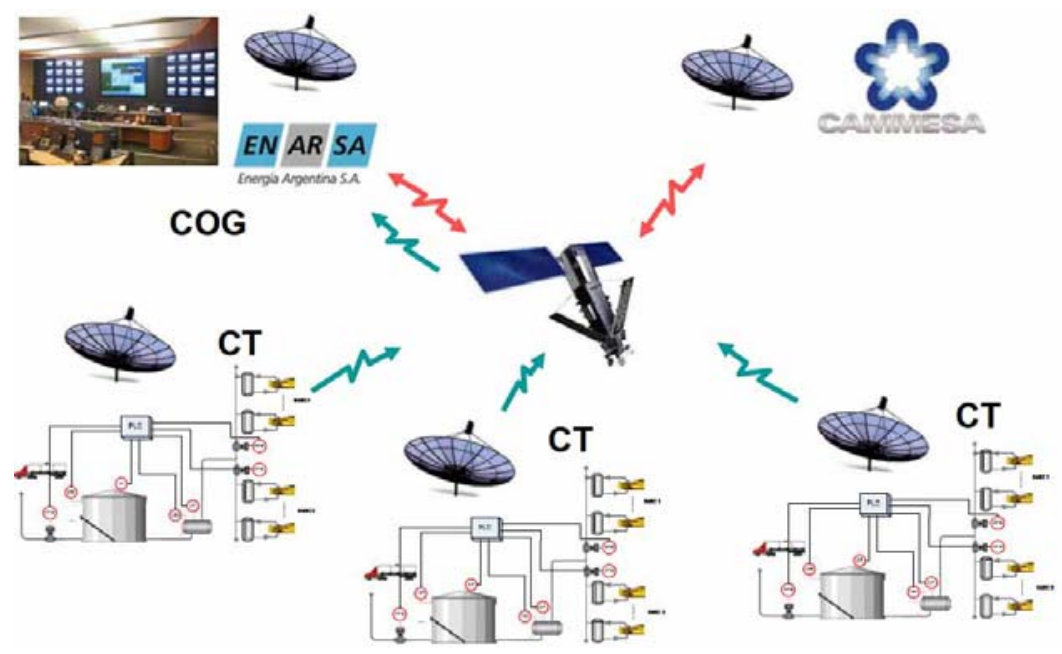

Fig. 7. Comunicaciones CAMMESA - COG - CT

\subsection{Arquitectura de las comunicaciones del SIS}

El esquema de comunicaciones del SIS, se divide en tres niveles de comunicaciones, los cuales serán explicados a continuación.

3.3.1. Comunicaciones entre los dispositivos inteligentes de campo y los tableros de E/S distribuidas o el tablero de PLC

Se entiende a un dispositivo inteligente de campo a cualquier equipamiento con capacidad de comunicarse mediante un puerto de comunicaciones a través de un bus de campo. Los protocolos pueden ser HART, MODBUS, PROFIBUS, entre otros.

En aquellos casos en que las distancias de cableado sean menores a 90 metros, se utilizará una conexión IEEE 802.3 - CSMA/CD (Ethernet). El cableado debe cumplir con los siguientes estándares y homologaciones:

- Estándar EIA/TIA-568, categoría 5e. 
- $\quad$ Norma IEC 11801/EN 50173, clase D.

Como alternativa, y en función de las complejidades que puedan presentarse (distancias de cableado, disponibilidad de canalizaciones existentes, costos de instalación), se puede utilizar un enlace inalámbrico WiFi para la comunicación entre los tableros de E/S distribuidas y el tablero de PLC. En este caso, el enlace WiFi deberá cumplir con las especificaciones del estándar IEEE 802.11.

3.3.2 Comunicaciones entre los tableros de E/S distribuidas y el tablero de PLC

Deberán ser MODBUS TCP/IP, ya que tanto el PLC de control como los módulos de E/S distribuidas soportan este protocolo. Además resulta simple para administrar y expandir. No se requiere usar herramientas de configuración complejas cuando se añade una nueva estación a una red MODBUS/TCP.

Se propuso la utilización de una conexión IEEE 802.8 - FDDI (fibra óptica), debido a que la finalidad de los tableros de E/S distribuidas es concentrar las señales de un conjunto de buses de campo alejados del tablero de PLC. Por ello, en virtud de las grandes distancias (mayores a 100 [m]) que generalmente separan al tablero de PLC de los tableros de E/S distribuidas, se establece la utilización de FDDI en lugar de cable de tipo par trenzado.

\subsubsection{Comunicaciones entre el tablero de PLC y el COG}

La información de los distintos sistemas (SMC, energía, etc.) almacenados en el PLC de control serán informados al COG de ENARSA, a través de un sistema de comunicaciones provisto por ENARSA. El sistema de comunicaciones finaliza en una conexión Ethernet en cada una de las CT, a la que debe vincularse el tablero de PLC.

\section{Conclusiones y trabajos futuros}

El desarrollo de este trabajo llevado a cabo por el LSC implicará una serie de beneficios de índole técnicos y económicos para la empresa ENARSA.

En el caso concreto del SMC líquido propuesto por el LSC. Este contempla una serie de ventajas económicas (ver 3). Dentro de estas ventajas se espera una reducción de los costos de inspección de hasta un $50 \%$ debido a la simplificación de las tareas y la disminución de los tiempos para llevarlas a cabo.

Estos beneficios implicarían para la empresa un ahorro de dinero que permite una amortización del costo del SMC en, a lo sumo, cinco años según estudios realizados por el LSC. Además, considerando una vida útil de las CT de cómo mínimo diez años, la aplicación del sistema SMC propuesto resulta técnica y económicamente viable.

Por otra parte, la implementación de la topología de la red de comunicaciones propuesta para telesupervisar las CT, permitirá facilitar a sus operarios las tareas de mantenimiento y detección de fallas en el sistema, debido a la minimización de componentes que integran la red. A la vez que se consigue un sistema confiable, de adecuada velocidad de transmisión de datos para su reporte en tiempo real y de fácil escalabilidad. 
En líneas generales, la instalación del sistema de supervisión (tanto local como remota) de la CT, conllevará a las siguientes ventajas:

- Reducción de los costos de inspección del combustible líquido.

- Eliminación de la incertidumbre del STOCK de combustible líquido.

- Mejor control y gestión de la logística, por parte de la empresa ENARSA, del combustible líquido suministrado a las CT.

- Cálculo del heat rate de los grupos generadores, y a partir del mismo determinar posibles fallas en el funcionamiento de los turbogeneradores.

La topología de red diseñada garantiza un sistema con alto nivel de disponibilidad, baja tasa de falla, flexible, aceptable velocidad de transferencia de datos y fácil adaptación de equipos y dispositivos con diversos tipos de enlace físico y protocolos de comunicación. La elección de MODBUS como protocolo principal a utilizar en las redes de comunicaciones de cada una de las CT (protocolo abierto y gratuito) en su versión TCP/IP, permite obtener velocidades de transmisión de datos con del orden del mseg.

Los trabajos futuros tendrán en cuenta el análisis de la información proveniente del COG (ej. Alarmas en tiempo real), en lo se denomina "análisis de tiempo diferido". El mismo permitirá estudiar en profundidad las contingencias que se producen en las CT, con el objetivo de mitigarlas o eliminarlas. Finalmente, es importante remarcar que las características técnicas tanto de la arquitectura de los sistemas de comunicaciones como también de las especificaciones técnicas del SMC correspondientes a las CT, otorgan al sistema de telesupervisión una amplia flexibilidad y posibilidades de expansión. Del mismo modo que garantizan una notable optimización de los recursos y máxima eficiencia en su desempeño. Todo ello, contribuye al desarrollo de un sistema de control y monitoreo de las CT de vanguardia en el ámbito nacional, con perspectivas de poder ser transferido y readecuado para su apliacación en otras centrales o industrias que posean procesos similares.

\section{Agradecimientos}

Los autores agradecen a la empresa ENARSA por haber depositado su confianza en el LSC para participar activamente en el desarrollo de este trabajo, el cuál se realiza en el marco de un convenio de mutua colaboración entre ambos.

\section{Referencias}

1. Comisión Nacional de Energía Atómica (CNEA). Síntesis del mercado eléctrico mayorista de la Republica Argentina. (2011).

2. Mantilla González, J. M.; Duque Daza C. y Galeano Urueña, C. "Distributed generation scheme analysis as an option for colombian electrical system". Rev. Fac. Ing. Univ. Antioquia N. ${ }^{\circ}$ 44. pp. 97-110. Junio, (2008). 
3. Sánchez, M.; Garduño, R.. y Chávez, R.. "Tendencias tecnológicas para el mejoramiento del desempeño de centrales de ciclo combinado". Boletín IIE, noviembre-diciembre del 2001, Instituto de Investigaciones Eléctricas, (2001).

4. Kuwar, R. ; Dewal, M. L. y Saini, K. "Utility of SCADA in Power Generation and Distribution System". Third IEEE International Conference on Computer Science and Information Technology (ICCSIT), pp 648-652 (2010).

5. Lakhoua, M. N. "SCADA applications in thermal power plants". International Journal of the Physical Sciences, vol. 5, pp. 1175-1182, (2010).

6. Sánchez, M. y Vite-Hernández, R.. "Use of a Rule-Based System for Process Control: Flow Instabilities in Axial Compressors Case Study". Instituto de Investigaciones Eléctricas (IIE) - Gerencia de Control e Instrumentación. C.A. Coello Coello et al. (Eds.): MICAI 2002, LNAI 2313, pp. 494-505, (2002).

7. Zhang, J. H.; Hao, J. H.; Hou, G. L. y An, X. M. "A Control Strategy for AGC systems in Communication Networked Environment”. Electric Utility Deregulation and Restructuring and Power Technologies, pp 2166-2170, (2008).

8. Ke, J.Y.; Tang, K.S.; Man, K.F.; Luk, P.C.K.; "Hierarchical genetic fuzzy controller for a solar power plant". IEEE International Symposium on Industrial Electronics. Proceedings. ISIE, pp 584 - 588 vol.2, (1998).

9. Bernard, J.A.. "Use of a Rule-Based System for Process Control”. IEEE Control Systems Magazine, pp 3-13, vol. 8, (1998).

10. Moghavvemi, M.; Yang, S.S. y Kashem, M.A. "A Practical Neural Network Approach for Power Generation Automation". International Conference on Energy Management and Power Delivery. Proceedings of EMPD pp 305-310 vol. 1, (1998).

11. Blas, L., Domínguez, D.; Furlani, R.; Ibarra, F.; López, D. ; Regalini, R. y Torres, J. L. "Criterios de relevamiento y actualización de las instalaciones para un SCADA de LMT rural". CIDEL Argentina (2006).

12. Regalini, R. H.; D. Domínguez, R. A. ; Furlani, F. ; Ibarra, D. C. ; López y J. L. Torres. "Redes de comunicaciones para el control de LMT rurales". CIDEL Argentina (2006).

13. Cobelo, F. "Arquitecturas abiertas de comunicación para la automatización de subestaciones”. En Nota Técnica p+c (2003). 